\title{
A CLASS OF MULTIVALENT FUNCTIONS WITH ASSIGNED ZEROS
}

TOSHIO UMEZAWA

1. Introduction. Recently A. W. Goodman $[1 ; 2]$ has studied the following two classes of multivalent functions:

(i) $p$-valently starlike functions denoted by $S(p)$ : A function $f(z)$ is said to be $p$-valently starlike with respect to the origin for $|z|<1$ if (a) $f(z)$ is regular and $p$-valent for $|z|<1$ and (b) if there exists a $\rho$ such that, for each $r$ in $\rho<r<1$, the radius vector joining the origin to $f\left(r e^{i \theta}\right)$ turns continuously in the counterclockwise direction and makes $p$ complete revolutions as $\theta$ varies from 0 to $2 \pi$.

(ii) Typically-real functions of order $p$ denoted by $T(p)$. A function

$$
f(z)=\sum_{n=0}^{\infty} b_{n} z^{n}
$$

is said to be typically-real of order $p$ if in (1.1) the coefficients $b_{n}$ are all real and if $f(z)$ is regular in $|z| \leqq 1$ and $\Im f\left(e^{i \theta}\right)$ changes sign $2 p$ times as $\theta$ traverses the boundary of the unit circle.

Concerning the above classes of functions he obtained the following results:

Let

$$
f(z)=z^{q}+\sum_{n=q+1}^{\infty} a_{n} z^{n}
$$

be a function of the set $S(p)$ or $T(p)$. Suppose that in addition to the $q$ th order zero at $z=0$, the function $f(z)$ has exactly $p-q$ zeros, $\beta_{1}, \beta_{2}, \cdots, \beta_{p-q}$, such that $0<\left|\beta_{j}\right|<1, j=1,2, \cdots, p-q$. Then

$$
\left|a_{n}\right| \leqq A_{n}, \quad n=q+1, q+2, \cdots
$$

where $A_{n}$ is defined by

$$
\begin{aligned}
F(z) & =\frac{z^{q}}{(1-z)^{2 p}} \prod_{j=1}^{p-q}\left(1+\frac{z}{\left|\beta_{j}\right|}\right)\left(1+z\left|\beta_{i}\right|\right) \\
& =z^{q}+\sum_{n=q+1}^{\infty} A_{n} z^{n} .
\end{aligned}
$$

The inequality (1.3) is sharp.

Received by the editors January 30, 1952. 
For functions of the set $T(p)$ he has obtained a more general result $[2 ; 3]$. However even that result cannot include the above result for $S(p)$ since in $S(p)$ the coefficients can be complex.

Now in the present paper we shall introduce a wider class of functions $D(p)$ which includes $S(p), T(p)$ and others in the case where $f(z)$ has $p$ zeros, proving that the inequality (1.3) is also valid for the functions of this class.

\section{Preliminary considerations.}

LEMma 1. Let

$$
w=f(z)=\sum_{n=0}^{\infty} a_{n} z^{n}
$$

be regular for $|z| \leqq 1$ and have $p(\geqq 0)$ zeros in $|z| \leqq 1$. Then there exists a point $\zeta(|\zeta|=1)$ for which the following equality holds

$$
\arg f(-\zeta)=\arg f(\zeta)+p \pi
$$

Proof. Without loss of generality, let $\arg f(-1)-\arg f(+1)<p \pi$. If a point $\zeta$ moves from +1 to $-1, \arg f(-\zeta)-\arg f(\zeta)$ varies continuously from arg $f(-1)-\arg f(+1)<p \pi$ to $2 p \pi-(\arg f(-1)$ $-\arg f(+1))>p \pi$, since $f(z)$ has $p$ zeros. Hence at a point $\zeta$ the equality (2.2) holds.

The special cases of Lemma 1 and the following Definition 1 we owe to N. G. DeBruijn [4] and S. Ozaki [5].

Definition 1. Let us say the diametral line of $f(z)$ for the straight line $[f(\zeta) 0 f(-\zeta)]$ when $\zeta$ satisfies Lemma 1.

Accordingly we have the following:

Lemma $1^{\prime}$. Let (2.1) be a function regular for $|z| \leqq 1$. Then there exists at least one diametral line of $f(z)$ in the w-plane.

Definition 2. Let $f(z)$ be regular for $|z| \leqq 1$ and let $C$ be the image curve of $|z|=1$. If $C$ is cut by a straight line passing through the origin in $2 p$, and not more than $2 p$ points, then $f(z)$ is said to be starlike of order $p$ in the direction of the straight line. Especially when the direction of starlikeness of order $p$ is that of the diametral line of $f(z), f(z)$ is said to belong to the class $D(p)$.

The idea of being starlike in one direction was introduced by M. S. Robertson [6] and also extended to general $p$ by him $[7 ; 8]$. And $D(1)$ was studied in $[4 ; 5]$.

Lemma 2. Let $f(z)=\sum_{n=0}^{\infty} a_{n} z^{n}$ be a member of the class $D(p)$. Further

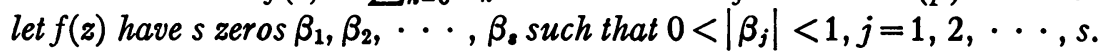


Then the function $F(z)$ defined by

$$
F(z)=f(z) g(z), \quad g(z)=z^{8} / \prod_{i=1}^{s}\left(z-\beta_{i}\right)\left(1-\bar{\beta}_{i} z\right)
$$

is also a member of the class $D(p)$.

Proof. Regularity of $F(z)$ in $|z| \leqq 1$ is evident. Now we easily see that

$$
g\left(e^{i \theta}\right)=1 / \prod_{j=1}^{s}\left|e^{i \theta}-\beta_{j}\right|^{2}
$$

Hence $\arg F\left(e^{i \theta}\right)=\arg f\left(e^{i \theta}\right)$ for every $\theta$. Consequently if $f(z) \in D(p)$, then $F(z) \in D(p)$.

3. The main theorem.

THEOREM 1. Let

$$
f(z)=z^{q}+\sum_{n=q+1}^{\infty} a_{n} z^{n}
$$

be a function of the set $D(p)$. Suppose that in addition to the qth order zero at $z=0$, the function $f(z)$ has exactly $p-q$ zeros, $\beta_{1}, \beta_{2}, \cdots, \beta_{p-q}$, such that $0<\left|\beta_{j}\right|<1, j=1,2, \cdots, p-q$. Then

$$
\begin{array}{rlr}
\left|a_{n}\right| & \leqq B_{n}, & n=q+1, q+2, \cdots, \\
\left|f\left(r e^{i \theta}\right)\right| & \leqq F(r) & \text { for } r<1,
\end{array}
$$

where $B_{n}$ and $F(r)$ are defined by

$$
\begin{aligned}
F(z) & =\frac{z^{q}}{(1-z)^{2 p}} \prod_{j=1}^{p-q}\left(1+\frac{z}{\left|\beta_{j}\right|}\right)\left(1+z\left|\beta_{j}\right|\right) \\
& =z^{q}+\sum_{n=q+1}^{\infty} B_{n} z^{n}
\end{aligned}
$$

Proof. Let us put

$$
E(z)=f(z) \cdot z^{p-q} / \prod_{i=1}^{p-q}\left(z-\beta_{i}\right)\left(1-\bar{\beta}_{i} z\right) .
$$

Then by Lemma $2, E(z) \in D(p)$ since $f(z) \in D(p)$, and

$$
\begin{aligned}
(-1)^{p-q} \prod_{i=1}^{s} \beta_{i} E(z) & =z^{p}+\alpha_{p+1} z^{p+1}+\cdots \\
& =\psi(z) \in D(p) .
\end{aligned}
$$


We wish now to show that

$$
\psi(z) \ll z^{p} /(1-z)^{2 p} .
$$

For the purpose it will be sufficient to assume that the diametral line in whose direction $\psi(z)$ is starlike of order $p$ is $\psi(1) 0 \psi(-1)$, since in the other cases we may consider $\psi(\zeta z)=g(z)$ for which $g(1) 0 g(-1)$ is the diametral line.

Let $\psi(1)=\omega=|\omega| e^{-i \alpha}$; then by our hypothesis

$$
\begin{array}{cc}
\Im e^{i \alpha} \psi\left(e^{i \theta}\right)>0 & \text { for } \theta_{28-1}<\theta<\theta_{2 s}, \\
\Im e^{i \alpha} \psi\left(e^{i \theta}\right)<0 & \text { for } \theta_{2 s}<\theta<\theta_{2 s+1}, \\
s=1,2, \cdots, p, \theta_{2 p+1}=\theta_{1}+2 \pi, \theta_{1}=0, \theta_{j}=\pi, 1<j \leqq 2 p .
\end{array}
$$

Let

$$
\phi(z)=(-1)^{p-1} \exp \left(-\frac{i}{2} \sum_{s=1}^{2 p} \theta_{s}\right) \cdot \prod_{s=1}^{2 p}\left(e^{i \theta_{0}}-z\right) / z^{p}
$$

then

$$
\phi\left(e^{i \theta}\right)=-2^{2 p} \prod_{s=1}^{2 p} \sin \frac{\theta_{e}-\theta}{2} .
$$

Hence we obtain

$$
\begin{aligned}
& \phi\left(e^{i \theta}\right)>0 \text { for } \theta_{2 s-1}<\theta<\theta_{2 s,} \\
& \phi\left(e^{i \theta}\right)<0 \text { for } \theta_{2 s}<\theta<\theta_{2 s+1}, \quad s=1,2, \cdots, p .
\end{aligned}
$$

Let

$$
G(z)=-i e^{i \alpha} \psi(z) \phi(z)=e^{i \beta}+\sum_{n=1}^{\infty} \gamma_{n} z^{n}
$$

then $G(z)$ is regular for $|z| \leqq 1$ and

$$
\Re G\left(e^{i \theta}\right) \geqq 0 \text {. }
$$

Accordingly by the principle of minimum for regular harmonic functions

$$
\Re G(z)>0 \quad \text { for }|z|<1 .
$$

Hence by Carathéodory's theorem

$$
\left|\gamma_{n}\right| \leqq 2 \Re e^{i \beta} \leqq 2 \quad \text { for } n=1,2, \cdots .
$$

Consequently

$$
G(z) \ll(1+z) /(1-z) .
$$


On the other hand from (3.11) we have

$$
\begin{aligned}
\psi(z)= & i e^{-i \alpha}(-1)^{p} \exp \left(\frac{i}{2} \sum_{s=1}^{2 p} \theta_{s}\right) \\
& \cdot z^{p} G(z) /\left\{\left(1-z^{2}\right) \prod_{s \neq 1, j}^{2 p}\left(e^{i \theta_{s}}-z\right)\right\}
\end{aligned}
$$

which is dominated by

$$
z^{p}\left(\frac{1+z}{1-z}\right) \cdot \frac{1}{1-z^{2}} \cdot \frac{1}{(1-z)^{2 p-2}}=\frac{z^{p}}{(1-z)^{2 p}}
$$

since we have (3.12).

From (3.4) and (3.5), we have

$$
f(z)=\psi(z) \prod_{i=1}^{p-q}\left(z-\beta_{i}\right)\left(1-\bar{\beta}_{i} z\right) /\left(\prod_{i=1}^{p-q} \beta_{i} z^{p-q}\right)
$$

which is dominated by

$$
\frac{z^{p}}{(1-z)^{2 p}} \prod_{i=1}^{p-q}\left(1+\frac{z}{\left|\beta_{i}\right|}\right)\left(1+\left|\beta_{i}\right| z\right) \cdot \frac{1}{z^{p-q}}=F(z)
$$

since we have (3.13). Hence we obtain

$$
\left|a_{n}\right| \leqq B_{n}, \quad n=q+1, q+2, \cdots,
$$

and

$$
\left|f\left(r e^{i \theta}\right)\right| \leqq F(r) \quad \text { for } r<1 \text {. q.e.d. }
$$

4. A class of functions related to $D(p)$.

Definition 3. Let $w=f(z)$ be regular for $|z| \leqq 1$ and $C$ be the image curve of $|z|=1$. Let, further, $P$ be the orthogonal projection of $f\left(e^{i \theta}\right)$ onto a straight line. Then $P$ will move on the straight line both positively or negatively when $\theta$ varies from 0 to $2 \pi$. If $P$ changes its direction of movement $2 p$ times when $\theta$ varies from 0 to $2 \pi$, then $f(z)$ is said to be convex of order $p$ in the direction which is perpendicular to the straight line. This class of functions has recently been studied by M. S. Robertson [9].

Especially if, when we represent $f(z), z f^{\prime}(z)$ in the same plane, the straight line is parallel to a diametral line of $z f^{\prime}(z)$, then $f(z)$ is said to be a member of $F(p)$.

LEMMA 3. $f(z)$ is a member of the class $F(p)$ if and only if $z f^{\prime}(z)$ belongs to the class $D(p)$. 
Proof. This is a generalization of M. S. Robertson's lemma [6].

It is sufficient to prove the lemma in the case where the diametral line of $f(z)$ is the real axis, since in the other cases we may consider $e^{i \alpha} f(z)$ with a suitable choice for the real parameter $\alpha$.

Using the identity

$$
\Im\left\{z f^{\prime}(z)\right\}=-\partial \Re f(z) / \partial \theta \quad \text { for }|z|=1
$$

we see, under the hypothesis,

$$
\begin{array}{rr}
\Im\left\{z f^{\prime}(z)\right\}=-\partial \Re f\left(e^{i \theta}\right) / \partial \theta>0 & \text { for } \theta_{2 s-1}<\theta<\theta_{2 s,} \\
\Im\left\{z f^{\prime}(z)\right\}=-\partial \Re f\left(e^{i \theta}\right) / \partial \theta<0 & \text { for } \theta_{2 s}<\theta<\theta_{2 s+1}, \\
s=1,2, \cdots, p, \theta_{j}=\theta_{1}+\pi, \theta_{2 p+1}=\theta_{1}+2 \pi .
\end{array}
$$

Hence $f(z) \in F(p)$ if and only if $z f^{\prime}(z) \in D(p)$.

THEOREM 2. Let

$$
f(z)=z^{q}+\sum_{n=q+1}^{\infty} a_{n} z^{n}
$$

be a function of the set $F(p)$. Suppose that in addition to the $(q-1)$ th order critical points at $z=0$, the function $f(z)$ has exactly $p-q$ critical points $\alpha_{1}, \alpha_{2}, \cdots, \alpha_{p-q}$ such that $0<\left|\alpha_{j}\right|<1, j=1,2, \cdots, p-q$. Then

$$
\begin{array}{rlrl}
\left|a_{n}\right| & \leqq q C_{n} / n, \quad n=q+1, q+2, \cdots, \\
\left|f\left(r e^{i \theta}\right)\right| & \leqq q \int_{0}^{r} \frac{F(r)}{r} d r, & \text { for } r<1, \\
\left|f^{\prime}\left(r e^{i \theta}\right)\right| & \leqq q F(r) / r, & \text { for } r<1,
\end{array}
$$

where $C_{n}$ and $F(r)$ are defined by

$$
\begin{aligned}
F(z) & =\frac{z^{q}}{(1-z)^{2 p}} \prod_{j=1}^{p-q}\left(1+\frac{z}{\left|\beta_{j}\right|}\right)\left(1+z\left|\beta_{i}\right|\right) \\
& =z^{q}+\sum_{n=q+1}^{\infty} C_{n} z^{n}
\end{aligned}
$$

Proof. Since $f(z) \in F(p)$,

$$
\frac{1}{q} z f^{\prime}(z)=z^{q}+\frac{1}{q} \sum_{n=q+1}^{\infty} n a_{n} z^{n} \in D(p)
$$

by Lemma 3.

By using the main theorem we have (4.2) and (4.4). By integrating $f^{\prime}(z)$ along a radius we have, for $z=r e^{i 0}$, 
1952]

$$
\begin{aligned}
& \left|f\left(r e^{i \theta}\right)\right|=\left|\int_{0}^{z} f^{\prime}(z) d z\right| \leqq \int_{0}^{r}\left|f^{\prime}\left(r e^{i \theta}\right)\right| d r \leqq q \int_{0}^{r} \frac{F(r)}{r} d r \\
& \text { for } r<1 \text {, }
\end{aligned}
$$

which completes the proof.

5. Subclasses of $D(p)$ and $F(p)$.

CoROLlaRY 1. Let $f(z)$ in the form (3.1) be regular for $|z| \leqq 1$ and assigned with the same zeros as in Theorem 1. Suppose that $f(z)$ satisfies one of the following conditions:

(i) $\Re\left[z f^{\prime}(z) / f(z)\right]>0$ for $|z|=1$,

(ii) $f(1)=$ real, $f(-1)=$ real and $\Im f\left(e^{i \theta}\right)$ changes sign $2 p$ times on $|z|=1$,

(iii) $f(z) \in T(p)$.

Then (3.2) and (3.3) hold.

Proof. (i) Since there exists at least one diametral line of $f(z)$ by Lemma $1^{\prime}$, and since $f(z)$ is starlike of order $p$ in every direction by the fact that $\Re\left[z f^{\prime}(z) / f(z)\right]>0$ on $|z|=1$ and $f(z)$ has $p$ zeros in $|z|<1, f(z)$ is evidently starlike of order $p$ in the direction of the above diametral line.

(ii) In this case the diametral line of $f(z)$ is evidently the real axis and is starlike of order $p$ in this direction by our hypothesis, which proves the corollary by using the main theorem.

(iii) This is a direct consequence of the preceding (ii).

CoROllaRY 2. Let $f(z)$ in the form (4.1) be regular for $|z| \leqq 1$ and assigned with the same critical points as in Theorem 2. Suppose that $f(z)$ satisfies one of the following conditions:

(i) $1+\Re\left[z f^{\prime \prime}(z) / f^{\prime}(z)\right]>0$ for $|z|=1$.

(ii) $f^{\prime}(1)=$ real, $f^{\prime}(-1)=$ real, and $f(z)$ is convex of order $p$ in the direction of the imaginary axis.

(iii) In (4.1) the coefficients are all real and $f(z)$ is convex of order $p$ in the direction of the imaginary axis.

Then (4.2), (4.3), and (4.4) hold.

Proof. (i) By our hypothesis $z f^{\prime}(z)$ has $p$ zeros in $|z|<1$ and $\Re\left[z\left\{z f^{\prime}(z)\right\}^{\prime} /\left\{z f^{\prime}(z)\right\}\right]>0$ on $|z|=1$. Hence $z f^{\prime}(z)$ is starlike of order $p$ in every direction. Consequently $z f^{\prime}(z) \in D(p)$ by Corollary 1 adopting (i). Accordingly $f(z) \in F(p)$ by Lemma 3 .

(ii) By our hypothesis - $\partial \Re f(z) / \partial \theta$ changes sign $2 p$ times on $|z|$ $=1$. Accordingly $\Im\left\{g f^{\prime}(z)\right\}$ changes sign $2 p$ times on $|z|=1$ by 
Lemma 3. And $1 f^{\prime}(1)=$ real, $(-1) f^{\prime}(-1)=$ real. Hence $z f^{\prime}(z) \in D(p)$. Consequently $f(z) \in F(p)$.

(iii) This is a special case of (ii).

\section{REFERENCES}

1. A. W. Goodman, On the Schwarz-Christoffel transformation and p-valent functions, Trans. Amer. Math. Soc. vol. 68 (1950) pp. 204-223.

2. - Typically-real functions with assigned zeros, Proceedings of the American Mathematical Society vol. 2 (1951) pp. 349-357.

3. A. W. Goodman and M. S. Robertson, $A$ class of multivalent functions, Trans. Amer. Math. Soc. vol. 70 (1951) pp. 127-136.

4. N. G. DeBruijn, Ein satz ïber schlichte Funktionen, Neder. Akad. Wetensch. vol. 44 (1941) pp. 47-49.

5. S. Ozaki, On the theory of multivalent functions II, Science Reports of the Tokyo Bunrika Daigaku Sect. A vol. 4 (1941) pp. 45-87.

6. M. S. Robertson, Analytic functions starlike in one direction, Amer. J. Math. vol. 58 (1936) pp. 465-472.

7. - A representation of all analytic functions in terms of functions with positive real parts, Ann. of Math. vol. 38 (1937) pp. 770-783.

8. - The variation of the sign of $V$ for an analytic function $U+i V$, Duke Math. J. vol. 5 (1939) pp. 512-519.

9. - A coefficient problem for functions regular in an annulus, Bull. Amer. Math. Soc. Abstract 57-4-301.

Gumma University, MaEbashi, JaPAN 